\title{
DESIGN AND DEVELOPMENT OF A TELEMANIPULATED WELDING ROBOT WITH VISUAL AND HAPTIC FEEDBACK
}

\author{
P.Abhijith Reddy ${ }^{1}$, T.Dharniju Reddy ${ }^{2}$ \\ ${ }^{I}$ B.Tech Graduate, Department of Mechanical Engineering, K.L University, Andhra Pradesh, India \\ ${ }^{2}$ B.Tech Graduate, Department of Mechanical Engineering, K.L University, Andhra Pradesh, India
}

\begin{abstract}
In today's world a large proportion of human effort is replaced by robots most of this change is visible in the field of manufacturing industries where in many works previously done by human beings are performed by machines or robots with minimal human interface. The problem with these human operated robots is that the operator and the workplace are at completely different places and the operator has no idea of the workplace and has to operate the machine using devices such as joysticks without knowing how the machine is operating, so in our paper we wish to develop a tele manipulating welding robot which mimics the action of the operator and also is complemented by visual and haptic feedback where in the entire setup would be placed on a mobile base.
\end{abstract}

Keywords: Human Interface, Telemanipulation, Visual Feedback, Haptic Feedback. $* * *$

\section{INTRODUCTION}

Designing an industrial robot these days has become a complicated task mainly because most of the work to be done these days is in remote and hazardous regions where in a human operator cannot go to. Besides most of the work space or surrounding is changing continuously with respect to time or in other words it is dynamic in nature.

Presently in our work we would the case of a welding robot which has to perform welding operations in remote or inaccessible areas and has to be operated by a human operator. We intend to design a telemanipulation system for performing the above mentioned tasks. Besides developing a telemanipulation system we would also like to enhance its performance by equipping or complementing it with visual and haptic feedback.

Telemanipulation is a process in which certain action is conveyed or carried to a place from where it is actually happening. Any telemanipulation system consists of two different parts namely the master and slave manipulators. Both these manipulators are geometrically similar not necessarily of the same size or dimensions. A master manipulator is the one which is in under use by the operator it can be viewed analogous to an input device. The slave on the other hand is a manipulator which is near the workplace and it mimics the action of the master manipulator.

Haptics on the other hand can be viewed to robots in a way similar to how the human beings possess the sense of touch it helps the operator to feel the object just as if it is in front of him despite the object being far away and he is not the one touching it is the slave manipulator that is in contact or touching it. Visual feedback on the other hand is like eyes to the operator which help the operator in guiding the slave manipulator to target which in this case is a work piece.

\section{PROBLEM DESCRIPTION AND PROPOSED}

\section{SOLUTION}

Welding task in inaccessible locations is a highly challenging task as the operator cannot go near the workpiece as it may be located in inaccessible or hazardous area so firstly our first problem would be how to get the slave manipulator with end effector to target region so the only solution is that the manipulator should be mobile in nature i.e. it should easily move from one place to another under the control of the human operator.

Now another problem that occurs is that how to perform the welding operation with ease as if the operator is right in front of the workpiece despite the fact that it is not. One solution to this is tele manipulation where in the master manipulator would be in the control of the operator and the slave would be on a mobile platform. But the question is how to make sure that tele manipulation takes place this would be taken care using potentiometers which would be placed at key joints in master manipulator and are linked to corresponding servos placed at similar locations on the slave. The potentiometers regulate the voltage in these servos and hence help the slave mimicking the operation performed by the master manipulator which is under the control of the operator.

Now despite the presence of tele manipulation and mobile platform these two would only help in getting the manipulator to the target region and perform the welding but would not give the operator the ease of welding which he normally does get when the workpiece is right in front of him. So to provide the operator with the sense that he is present in the immediate surrounding of the work piece and also to improve the quality of the weld bead we also provide the manipulator with visual and haptic feedback. 


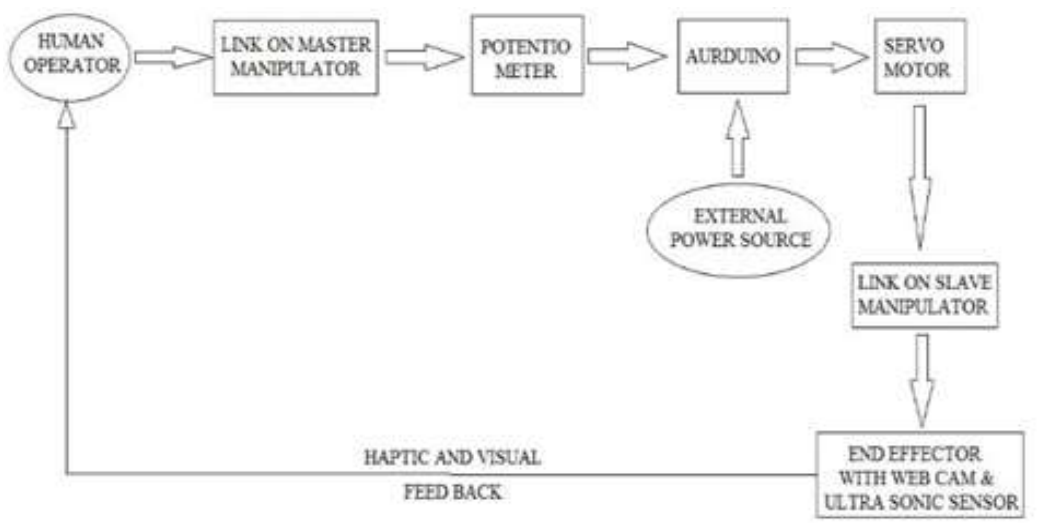

Fig 2.1 Block diagram of the proposed tele manipulating system setup for welding operation

\section{BUILDING THE PROTOTYPE}

\subsection{Master and Slave Manipulator}

The master manipulator links are made from acrylic sheets according to the required dimensions. The base is rotated using a servomotor which gives it a 180 degree rotation angle. On the base are two slotted grooves to mount two other servo motors of which one acts an idle motor i.e. It just supports the pair of lower links while other servo is used to rotate or control the motion of lower links on the other end of lower links there is a dual shaft servo motor placed which is intern used to control the upper links. The upper links support the electrode and an ultrasound sensor which is used as a part of haptic feedback system which coaxially moves with the electrode. Now the master manipulator consists of links geometrically similar to those on slave but made out of cardboard. The links are place in identical places as those placed in slave the only difference being that here instead of servos their position is occupied by potentiometers. Now the question arises how the tele manipulation occurs now as the links in the master are moved this intern rotates the knob on potentiometer which intern act as a variable resistor there by regulating the amount of voltage entering the servo using and Arduino board i.e. the Arduino board takes voltage from a power source like a laptop or power bank and the potentiometer meter sends only a fraction of the output voltage from Arduino to servo hence rotating the servo.

\subsection{Mobile Platform}

The slave manipulator is placed on a mobile base which consists of four tyres controlled by a 60rpm geared motor each. These motors are powered by a 12 volt battery and controlled by dpdt switches thus allowing us to reverse the polarity of the motors and allowing us to move back front left and right.

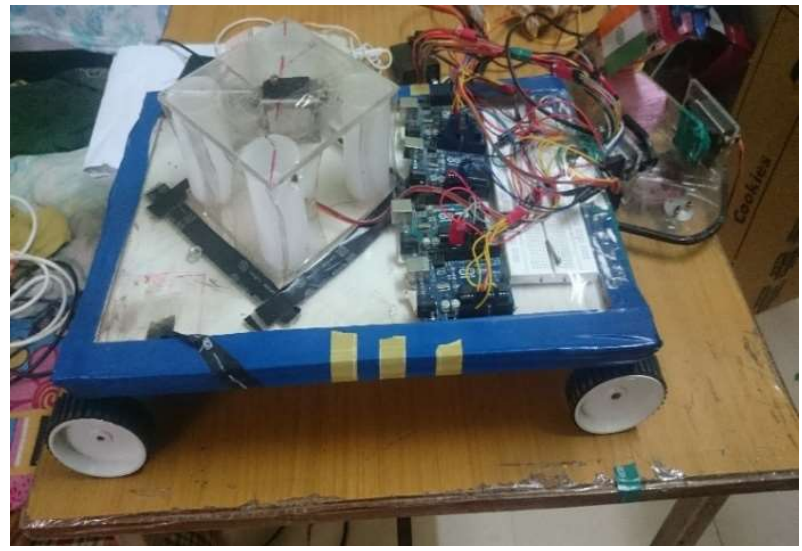

Fig 3.2.1 Mobile platform with the slave manipulator base

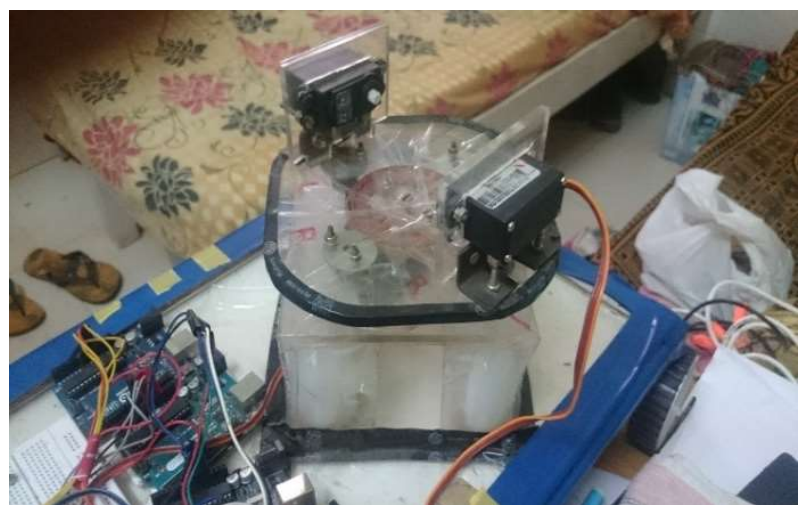

Fig 3.2.2 supports for lower link with servos

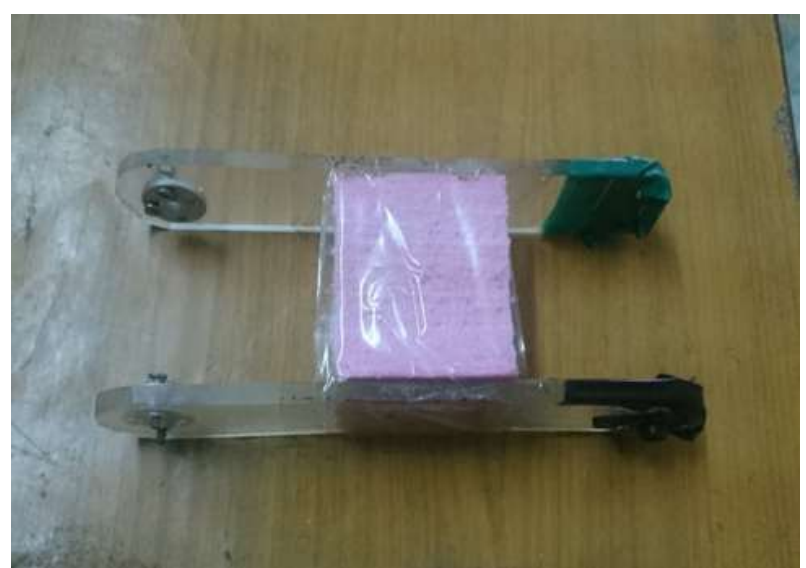

Fig 3.2.3 the lower link with thermos foam in between used as a spacer 


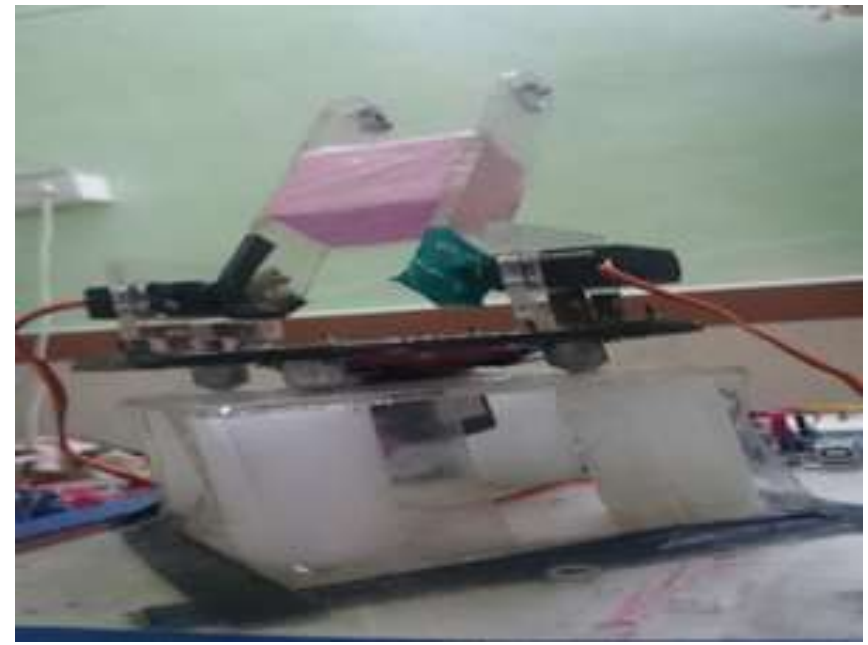

Fig 3.2.4 lower link placed in the supports

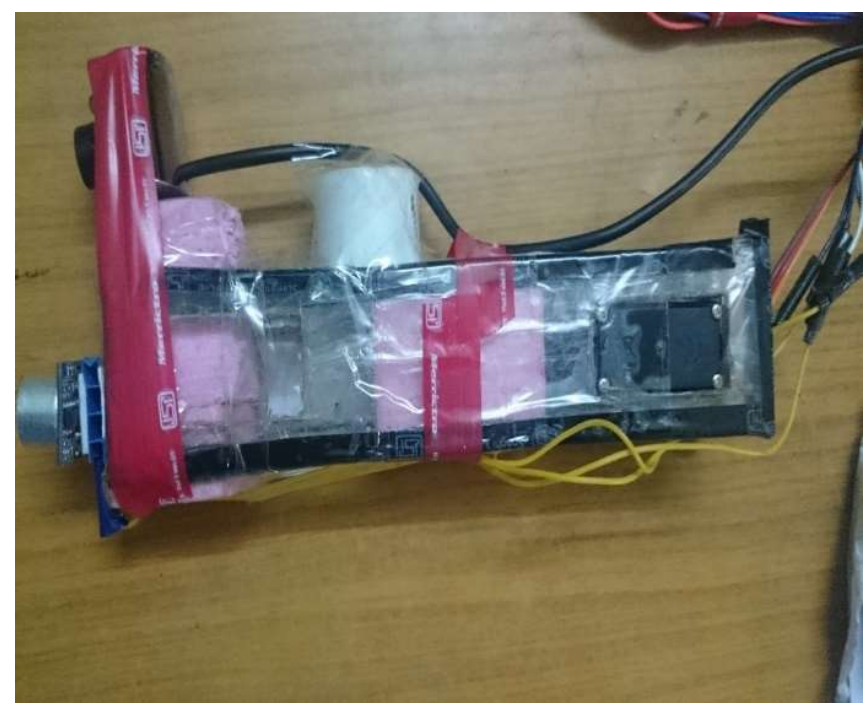

Fig 2.3.5 upper link or the end effector

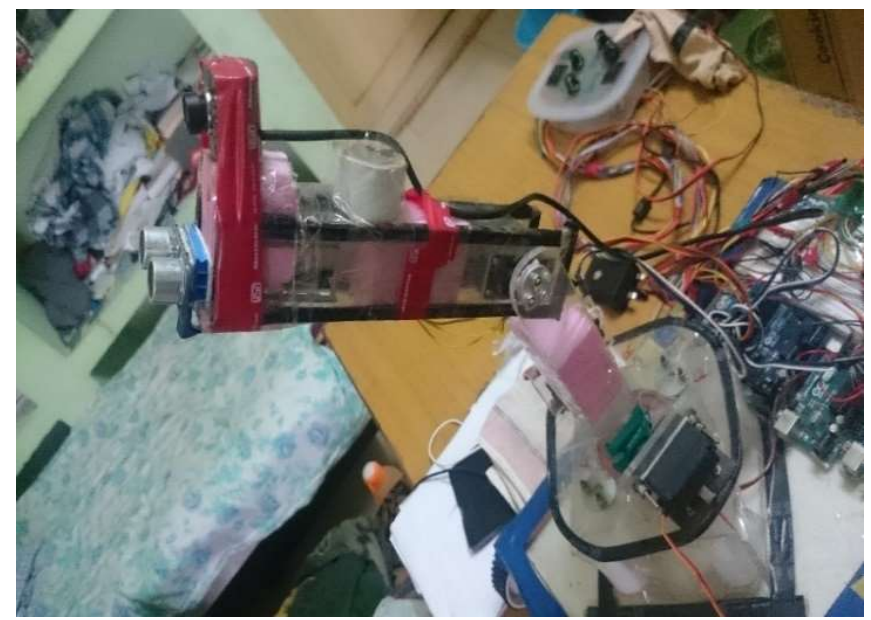

Fig 2.3.6 placement of end effector or upper link on top of the lower link

\subsection{Haptic Feedback}

The haptic feedback in our device makes use of a ultrasound sensor mounted on slave manipulator coaxial to the electrode this helps us in determining the distance between the electrode and the workpiece and using an Arduino board depending on the distance between the electrode and work piece it would activate a number of vibration motors mounted an glove worn on the hand of the operator thus giving him a faint tickling sensation giving him an idea of how far the electrode is from the workpiece further completing a sense of touch factor.

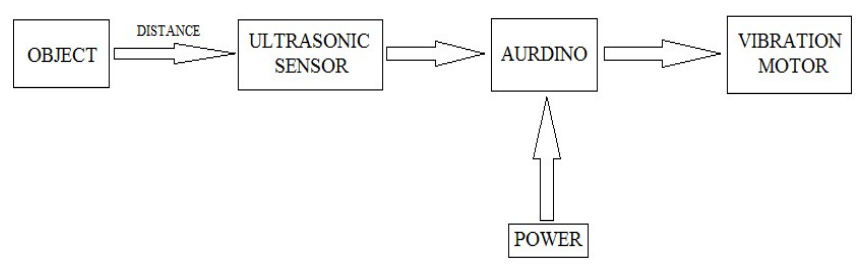

Fig 3.3.1 Block diagram of haptic Feedback system

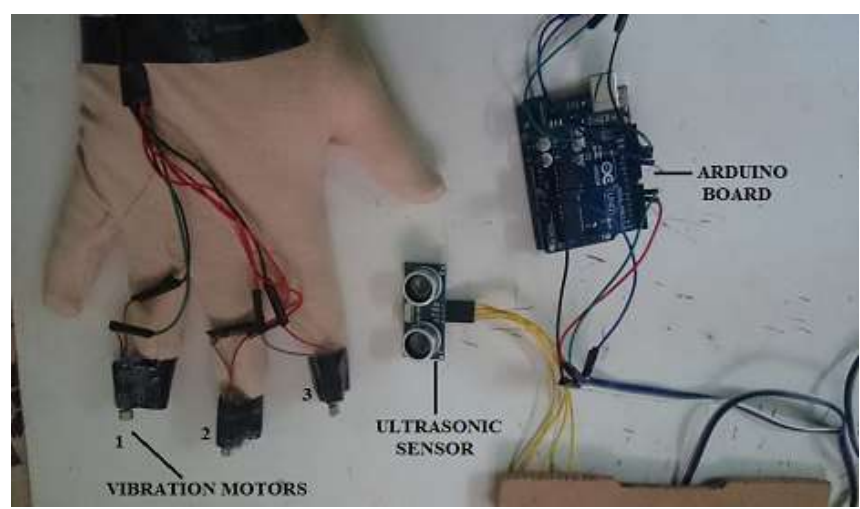

Fig 3.3.2 haptic feedback system

\subsection{Visual Feedback System}

The visual system in our manipulator consists of a web cam that is coaxially mounted on the end effector or the upper link the camera is placed above the electrode which in turn is placed above the ultrasound sensor.

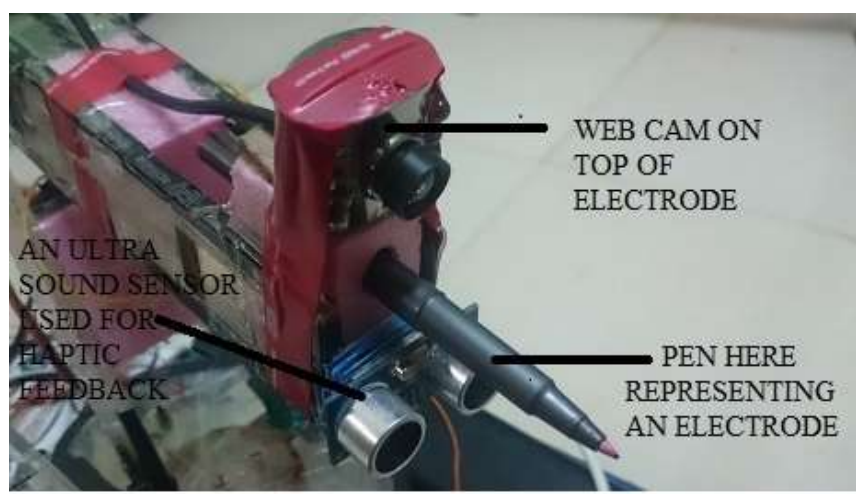

Fig 3.4 The end effector

\section{EXPERIMENTATION}

\subsection{Experiment to Check the Ability to Weld a}

\section{Corner Joint}

Objective:_To check whether the given slave manipulator can perform a vertical weldingOf a corner joint.

Experimental setup: The experimental setup consists of certain cardboard cartons and shoe boxes to represent a 
location which is inaccessible to human beings in this case the operator it initially starts with a narrow passage which extends into large space where in the workpiece is kept.

Procedure: The experimental setup as shown in the figures below was setup to represent atypical corner joint. The end effector consisted of a highlighter which represented an electrode the ink coming out of it would represent the weld bead. To represent the work Piece a paper was placed at the place in the form of a corner joint. In the experiment the mobile platform had overcome or had to pass through a narrow passage and reach the work piece and hence its mobility was tested its movement was controlled by the operator with the help of video or visual feedback coming from the camera placed on the end effector which would be visible on the laptop screen right in front of the operator using which the operator would guide the slave manipulator to its target or in the present case the corner joint.

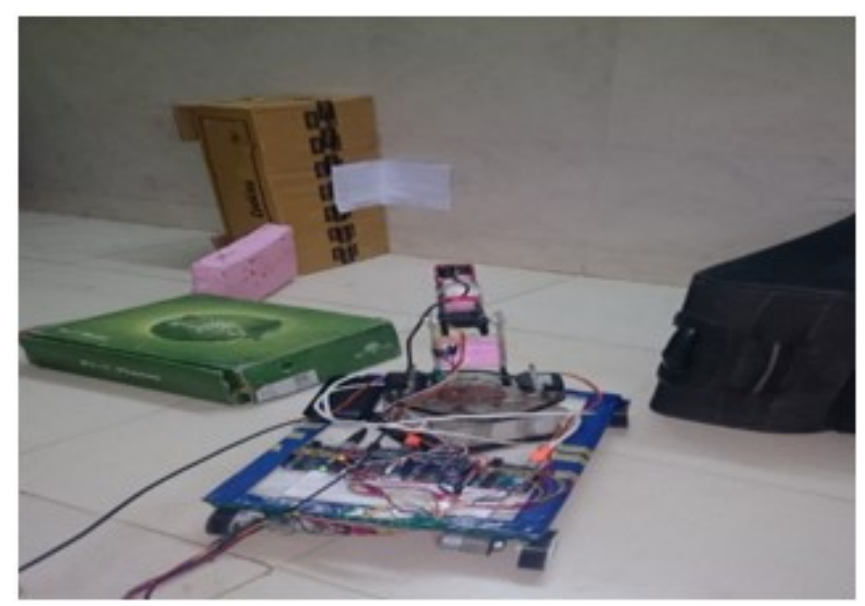

Fig 4.1.1: this figure shows the experimental setup where in the slave manipulator is just about to pass through the narrow passage. As you can see the paper the corner represents the workpiece.

\section{Observations}

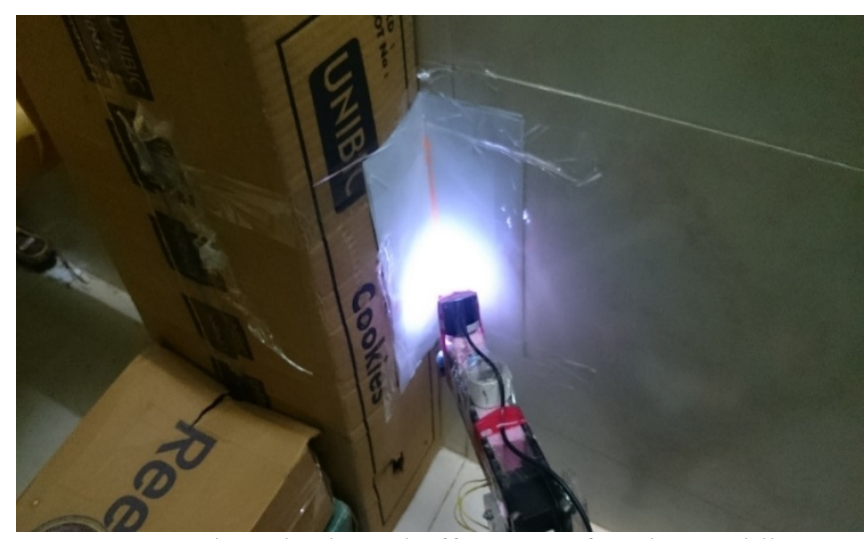

Fig 4.1.2: the robotic end effector performing welding

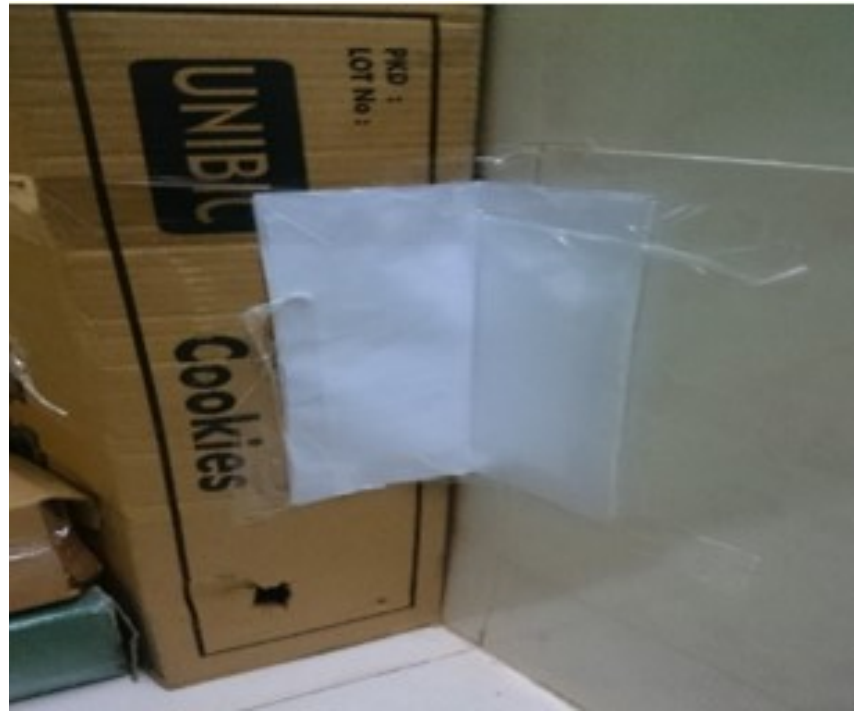

Fig 4.1.3 workpiece before welding

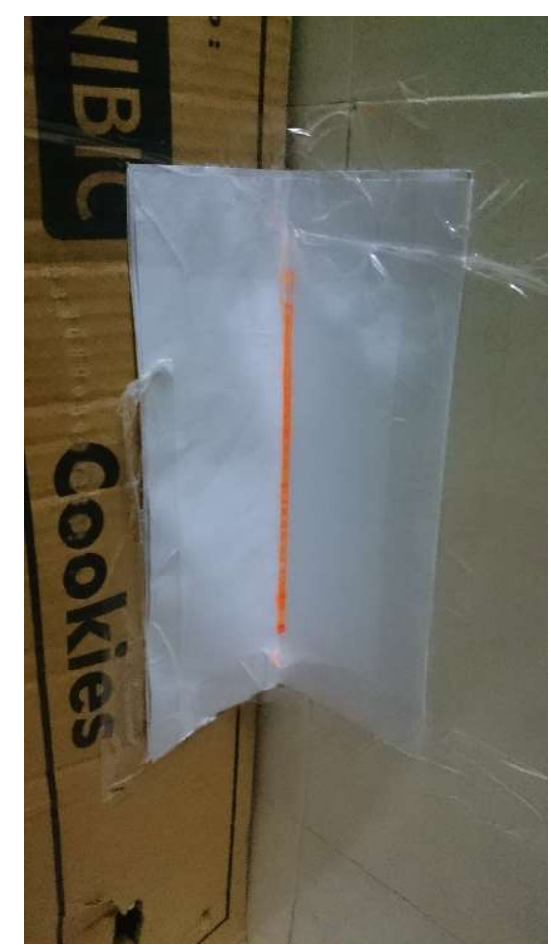

Fig 4.1.4 the weld bead after welding process indicated by the orange line

\subsection{Experiment to Check the Working of the Haptic}

\section{Feedback System}

Objective: To check the functioning of the haptic feedback system present on the slave manipulator of the tele manipulation system.

Experimental Setup: In this experiment the setup consists of a cardboard box representing a fake wall or workpiece. The box is placed right in front of the ultrasound sensor and the glove is worn by a human operator. Each of the motors on the glove were fixed with a card board piece of plus sign on it which when under operation would create an optical illusion. 


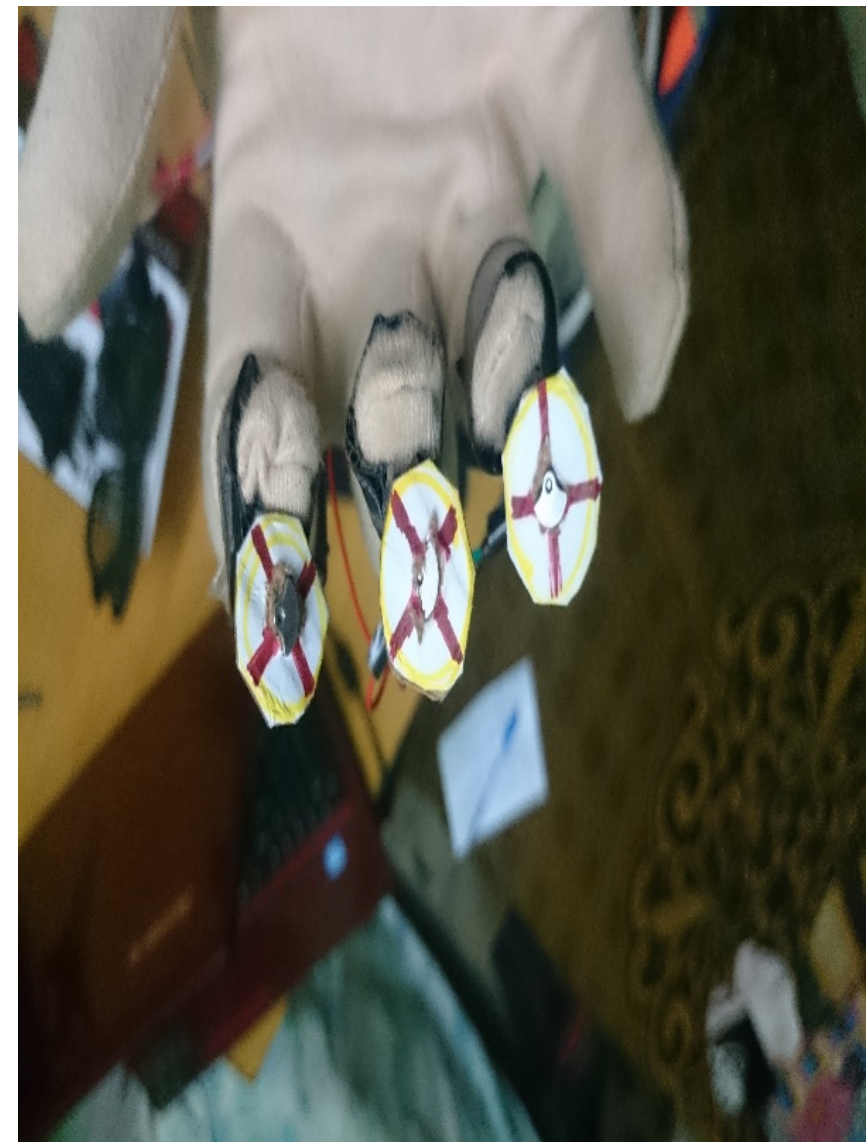

Fig 4.2.1 card board indicators on the vibration motors when they are stationary

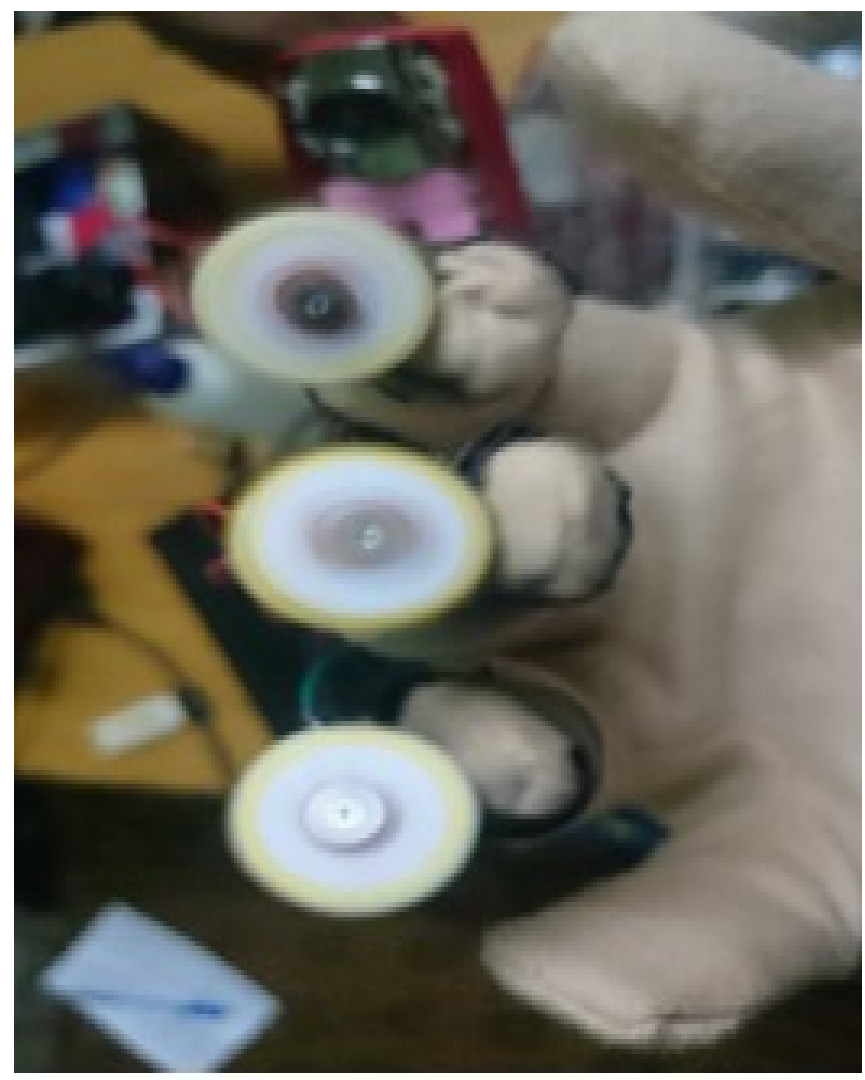

Fig 4.2.2 card board indicators creating optical illusion when all three motors are running

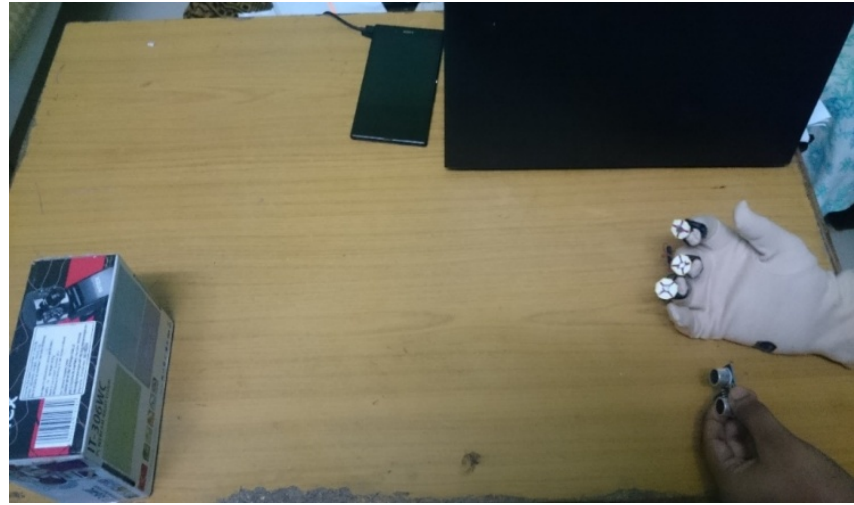

Fig 4.2.3 Experimental setup for testing haptic feedback system the ultrasound sensor in hand indicating position of electrode and the box indicating the workpiece

Procedure: Now as we know the box indicates the wall or work piece the box will be moved towards the sensor to simulate the movement of the end effector towards the workpiece. The system would be tested at a distance of $45 \mathrm{~cm}, 30 \mathrm{~cm}$ and $15 \mathrm{~cm}$ between the sensor and the workpiece the Arduino is programed that at a distance of $45 \mathrm{~cm}$ only one motor vibrates at $30 \mathrm{~cm}$ two motors would vibrate and three motors would vibrate at $15 \mathrm{~cm}$. As the distance between the workpiece and the electrode decreases the number of motors vibrating is supposed to increase and increase the tickling sensation to the operator hence completing the sense of touch or haptic feedback.

Observation: The experiment was performed at all the three different positions and observations were noted.

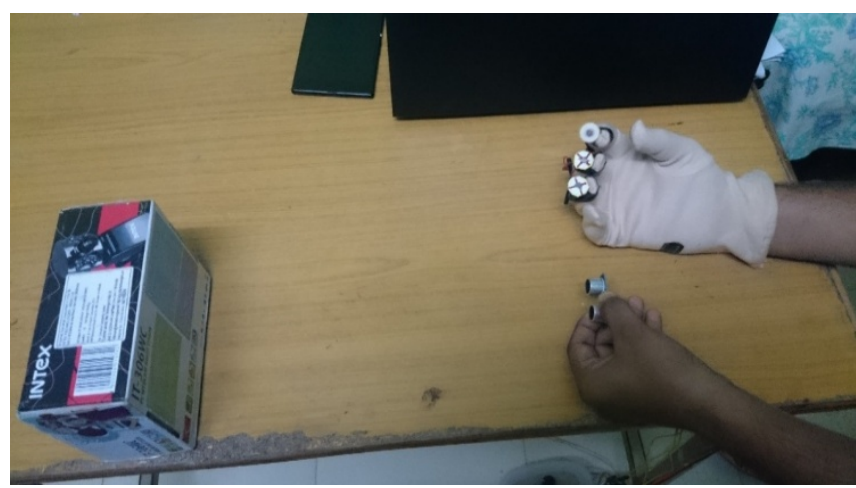

Fig 4.2.4 Experimental setup for $45 \mathrm{~cm}$ distance showing only one motor vibrating

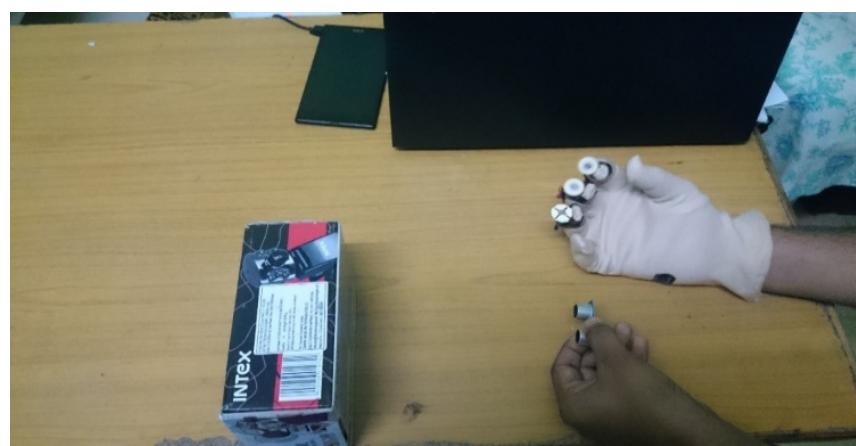

Fig 4.2.5 Experimental setup for $30 \mathrm{~cm}$ distance showing two motors vibrating 


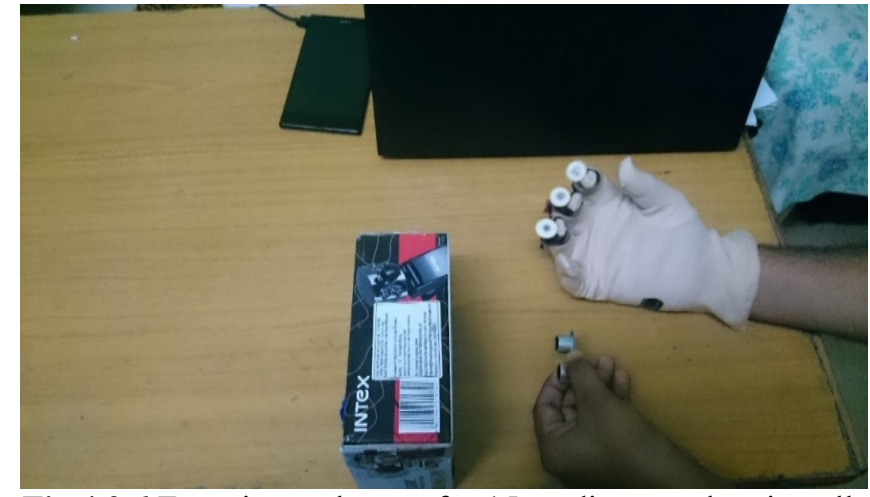

Fig 4.2.6 Experimental setup for $15 \mathrm{~cm}$ distance showing all three motors vibrating

\section{RESULTS AND DISCUSSIONS}

The first experiment resulted in a perfectly straight weld bead on the corner joint which confirmed the functioning of the mobile platform and also the slave manipulator along with the visual feedback.

The haptic feedback test was performed by using the experimental setup as shown in chapter four and as the distance between the electrode and the workpiece decreased the number of motors vibrating increased further increasing the sensation of touch or tickle and hence helping the operator.

The test was performed at distance of $15 \mathrm{~cm}$ at which 3 motors vibrated, At $30 \mathrm{~cm}$ when 2 motors vibrated and at 45 $\mathrm{cm}$ where in only one motor vibrated.

\section{CONCLUSION}

In the present project we were able to create a primitive haptic and visual feedback system. Certain improvements can be made especially in the way the motion is transmitted between were in which the action is conveyed using potentiometers and wires reducing the systems portability a wireless system for transmission of motion between master and slave manipulators can be thought of.

\section{FUTURE SCOPE}

The robotic tele-manipulation set-up developed in this project can be explored for being utilised in a remotely operated welding task based on Haptic and visual based feedback. It is expected that a human operator can maintain a constant arc gap based on the sensory feedback system, through the potentiometer based master controller.

The performance of the robotic set-up can be further improved by properly synchronising the master with the slave manipulator.

Another most important area on which future work can be carried would be on the field how to reduce the unnecessary vibrations produced in the potentiometers which actually cause the slave to move even without the operator actually meddling with it.

\section{REFERENCES}

[1] Claudio Melchiorri, "Robotic Tele-manipulation Systems: An Overview on Control Aspects" DEIS Dept. of Electronics, Computer Science and Systems University of Bologna - Italy, 1999.

[2] Barbagli, F. and Salisbury, K. (2003), "The effect of sensor/actuator asymmetries in haptic interfaces", Proceedings of the 11th Symposium on Haptic Interfaces for Virtual Environment and Teleoperator Systems, Los Angeles, CA, March 2003, pp. 140-7.

[3] R. Ellis, O. Ismaeil, and M. Lipsett, IDesign and evaluation of a high-performance haptic interface," Robotica, vol. 14, pp. $321\{327,1996$.

[4] Turner, M.L., Findley, R.P., Griffin, W.B., Cutkosky, M.R.,Gomez, D.H., 2000, "Development and Testing of aTelemanipulation System with Arm and Hand Motion,"Accepted to 2000 ASME IMECE Symp. on Haptic Interfaces.

[5] G. Hirzinger, B. Brunner, J. Dietrich, and J. Heindl, "Sensor- Based Space Robotics - ROTEX and Its Telerobotic Features," IEEE Trans. Robotics Automat., vol. 9, no. 5, 1993, pp. $649-663$.

[6] T. Yoshikawa and K. Yoshimoto, Haptic simulation of assembly operation in virtual environment," in Proc. ASME Dynamic Systems and Control Division, vol. 69-2, pp. $1191\{1198$, ASME, 2000.

[7] solarbotics.com/product/50450/

[8] http://www.robosoftsystems.co.in/roboshop/index.php/ motor-accessories/dc-motors-servo-motors/12v-dcgeared-motor-506.html

[9] http://letsmakerobots.com/node/30209

[10] http://www.futurlec.com/HardwareMain.shtml

[11] http://www.digikey.com/product-detail/en/eswitch/R5DBLKBLKEF0/EG1539-ND/301985.

[12] www.instructables.com

[13] https://www.youtube.com

[14] www.nskelectronics.com

\section{BIOGRAPHIES}

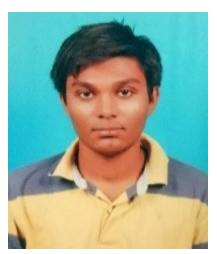

Mr.P.Abhijith Reddy has receivedhis B.techin mechanical engineering from K.L.University and is presently working in cyient ltd hyderabad as a trainee design engineer. Former member of kluasme student section.

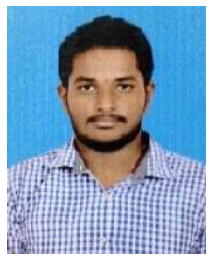

Mr.T.Dharniju Reddy has received his B.Tech in mechanical engineering from K.L.University. Former member of kluasme student section. 